\title{
The Binaural Interaction Component in Rhesus Macaques (Macaca mulatta)
}

\author{
John Peacock, ${ }^{1}$ Chase A. Mackey, ${ }^{2,4}$ Monica A. Benson, ${ }^{1}$ Jane A. Burton, ${ }^{2,4}$ Nathaniel T. Greene, ${ }^{3}$ \\ Ramnarayan Ramachandran, ${ }^{4}$ and ${ }^{-}$Daniel J. Tollin ${ }^{1}$
}

https://doi.org/10.1523/ENEURO.0402-21.2021

${ }^{1}$ Department of Physiology and Biophysics, University of Colorado School of Medicine, Aurora, CO 80045, ${ }^{2}$ Neuroscience Graduate Program, Vanderbilt University, Nashville, TN 37240, ${ }^{3}$ Department of Otolaryngology, University of Colorado School of Medicine, Aurora, CO 80045, and ${ }^{4}$ Department of Hearing and Speech Sciences, Vanderbilt University Medical Center, Nashville, TN 37212

\begin{abstract}
The binaural interaction component $(\mathrm{BIC})$ is a sound-evoked electrophysiological signature of binaural processing in the auditory brainstem that has received attention as a potential biomarker for spatial hearing deficits. Yet the number of trials necessary to evoke the BIC, or its measurability, seems to vary across species: while it is easily measured in small rodents, it has proven to be highly variable and less reliably measured in humans. This has hindered its potential use as a diagnostic tool. Further measurements of the BIC across a wide range of species could help us better understand its origin and the possible reasons for the variation in its measurability. Statistical analysis on the function relating BIC DN1 amplitude and the interaural time difference has been performed in only a few small rodent species, thus it remains to be shown how the results apply to more taxonomically diverse mammals, and those with larger heads. To fill this gap, we measured BICs in rhesus macaque. We show the overall behavior of the BIC is the same as in smaller rodents, suggesting that the brainstem circuit responsible for the BIC is conserved across a wider range of mammals. We suggest that differences in measurability are likely because of differences in head size.
\end{abstract}

Key words: auditory brainstem response; binaural hearing; binaural interaction component; macaque

\section{Significance Statement}

This article reports measurements of the binaural interaction component $(\mathrm{BIC})$ of the auditory brainstem response (ABR) in rhesus macaques. Comparison with other species reveals that the behavior of the macaque $\mathrm{BIC}$ is similar, and, based on data available so far, statistically indistinguishable to previously measured small rodents, suggesting that the brainstem circuit that generates it is largely conserved across all rodents and primates. Differences in the measurability of the BIC are likely because of variation in head size rather than differences in the neuroanatomy.

\section{Introduction}

The auditory brainstem response (ABR) is a non-invasive measure of auditory function recorded from scalp electrodes. ABRs comprise distinct peaks (five in humans, four in small mammals), which represent synchronized neural activity at nodes in the ascending pathway (Jewett et al., 1970). In humans, summing the monaural Waves I-

Received September 27, 2021; accepted November 25, 2021; First published December 1, 2021.

The authors declare no competing financial interests.
IV (Waves I-III in small mammals) approximates the amplitude of the binaural-evoked ABR, indicating that these waves are generated by monaural structures. However, binaural Wave $\mathrm{V}$ amplitude (Wave IV in small mammals) is less than the sum of the monaural waves, suggesting that neurons producing this wave are binaural. The residual waveform after subtracting the sum of the monaural from

Author contributions: J.P., R.R., and D.J.T. designed research; J.P., C.A.M., M.A.B., J.A.B., R.R., and D.J.T. performed research; J.P., C.A.M., N.T.G., and D.J.T. analyzed data; J.P. and D.T. wrote the paper. 
the binaural $A B R s$ is the binaural interaction component (BIC; Dobie and Berlin, 1979).

The BIC has received attention as an objective, noninvasive measure of binaural hearing ability. The amplitude and latency of its prominent negative peak, termed DN1, change systematically with the binaural cues to sound location: interaural differences in level (ILD) and time (ITD), and are also predictive of the perceived lateralization of a stimulus (Furst et al., 1990). Importantly, DN1 is reduced or completely absent in clinical populations that exhibit binaural hearing impairments (for review, see Laumen et al., 2016), including children with central auditory processing (Delb et al., 2003) and autism spectrum disorders (EIMoazen et al., 2020), as well as in individuals with early conductive (Gunnarson and Finitzo, 1991) and normal age-related hearing loss (Van Yper et al., 2016). Clinical detection of specific binaural hearing impairment has remained elusive as audibility is often unaffected in these populations; therefore, the BIC could serve to diagnose binaural deficits as well as delineate auditory dysfunction occurring at more peripheral brainstem levels from higher level cortical or cognitive impairment (e.g., autism and age-related hearing loss).

While the BIC holds potential as a diagnostic tool, its measurement is highly variable in humans (Sammeth et al., 2020). Understanding the specific brainstem circuits underlying the $\mathrm{BIC}$ could help explain its unreliable nature, improve methods for its measurement, and improve its clinical utility as a biomarker. Converging lines of evidence point to the lateral superior olive (LSO) as the circuit producing the BIC; comparative studies reveal that the $\mathrm{BIC}$ can be evoked in all mammalian species tested so far that possess an LSO [not all of which have a medial superior olive (MSO)] and computational studies report that LSO-like binaural excitation-inhibition processing can give rise to the BIC (Ashida et al., 2016; Benichoux et al., 2018).

The BIC has been measured in a small but growing number of species. Benichoux et al. (2018) have shown that the function relating BIC DN1 amplitude as a function of ITD is statistically the same across the tested species, suggesting conservation of the brainstem circuitry producing the BIC. However, this study only gathered data on small rodents with relatively small head sizes. Thus, it remains to be fully shown whether the results can be applied more generally across mammals, and to much larger species. In this study, we measured BICs in rhesus macaques (Macaca mulatta), a primate with a much larger head

This work was supported by National Institutes of Health grants F31 DC 019823-01A1 (C.A.M.), F32 DC 019817 (J.A.B.), R01-DC015988 (R.R.), and R01-DC011555 (D.J.T.)

Acknowledgements: We would like to thank Mary Feurtado for assistance with the use of anesthesia.

Correspondence should be addressed to John Peacock at john.2. peacock@cuanschutz.edu.

https://doi.org/10.1523/ENEURO.0402-21.2021

Copyright (C) 2021 Peacock et al.

This is an open-access article distributed under the terms of the Creative Commons Attribution 4.0 International license, which permits unrestricted use, distribution and reproduction in any medium provided that the original work is properly attributed. size than the rodents, but smaller than humans. We measured ABRs with varying ITD and examined how this compares to other species.

\section{Materials and Methods}

\section{Animal preparation}

Experimental procedures complied with guidelines set forth by the National Institutes of Health and approved by the Vanderbilt University Medical Center Animal Care and Use Committee. Eight normal hearing adult male rhesus macaques were used ranging in age from five to seven years and weighed $9-12 \mathrm{~kg}$. The average interaural diameter did not vary much across individuals, with the mean being $\sim 67$ $\mathrm{mm}$. Monkeys were anaesthetized with ketamine $(3-5 \mathrm{mg} /$ $\mathrm{kg})$ and dexmedetomidine $(5-15 \mu \mathrm{g} / \mathrm{kg})$. They were then placed in a sound treated booth (ER-247, Acoustic Systems) in a prone position. Vital signs were continuously monitored.

\section{Auditory Brainstem Response (ABR)}

Platinum subdermal needle electrodes (F-E2-12 electrodes, Grass Technologies) were placed at the apex along the interaural axis between the ears, with a reference electrode at the nape of the neck and ground on the animal's shoulder. Custom eartips were placed in the animals ears. The monaural and binaural ABR stimuli were designed in custom built MATLAB software (Benichoux et al., 2018). Stimuli were generated and evoked potentials recorded via an RME Fireface UCX soundcard (RME Audio). ABR potentials were collected via an WPI ISO-80 (World Precision Instruments) amplifier. Stimuli consisted of $90 \mathrm{~dB}$ SPL clicks presented at an average rate of 33/s. These were presented using TDT MF-1 speakers (TuckerDavis Technologies) via the eartips. The ITD of the binaural signal was varied between $\pm 1500 \mu$ s in steps of 500 $\mu \mathrm{s}$. A total of 3000 repetitions of the signal were presented, interleaved, for each stimulus condition, ITD, left ear monaural, and right ear monaural. Earphones were calibrated using a Bruel \& Kjær type 4182 probe microphone.

\section{Results}

Figure 1 shows $A B R$ waveforms across all eight monkeys. Figure $1 A$ shows averaged left ear $A B R s ; 1 B$ the right ear, and $1 C$ the binaural signal (ITD $=0 \mu \mathrm{s})$. Figure $1 D$ shows the sum of the two monaural responses; and $1 E$ shows the BIC. In all parts of the figure the shaded area shows the standard error of the mean, while the grey lines show the data from each individual monkey. ABR waveforms appeared consistent across all individuals. Averaged ABRs reveal peaks II, III and IV (wave I is difficult to see with a midline electrode montage), and the difference between the binaural and the sum of the monaural waveforms produces BIC DN1 with a latency of $\sim 4 \mathrm{~ms}$.

Figure $2 A-D$ shows the raw $\mathrm{BIC}$ data for each measured ITD. BICs for positive (left leading) and negative (right leading) ITDs are shown in the same panel. Grey lines show measurements from individual monkeys and black lines show the mean. BIC DN1 is visible at all ITDs, but the amplitude systematically diminishes and the latency 

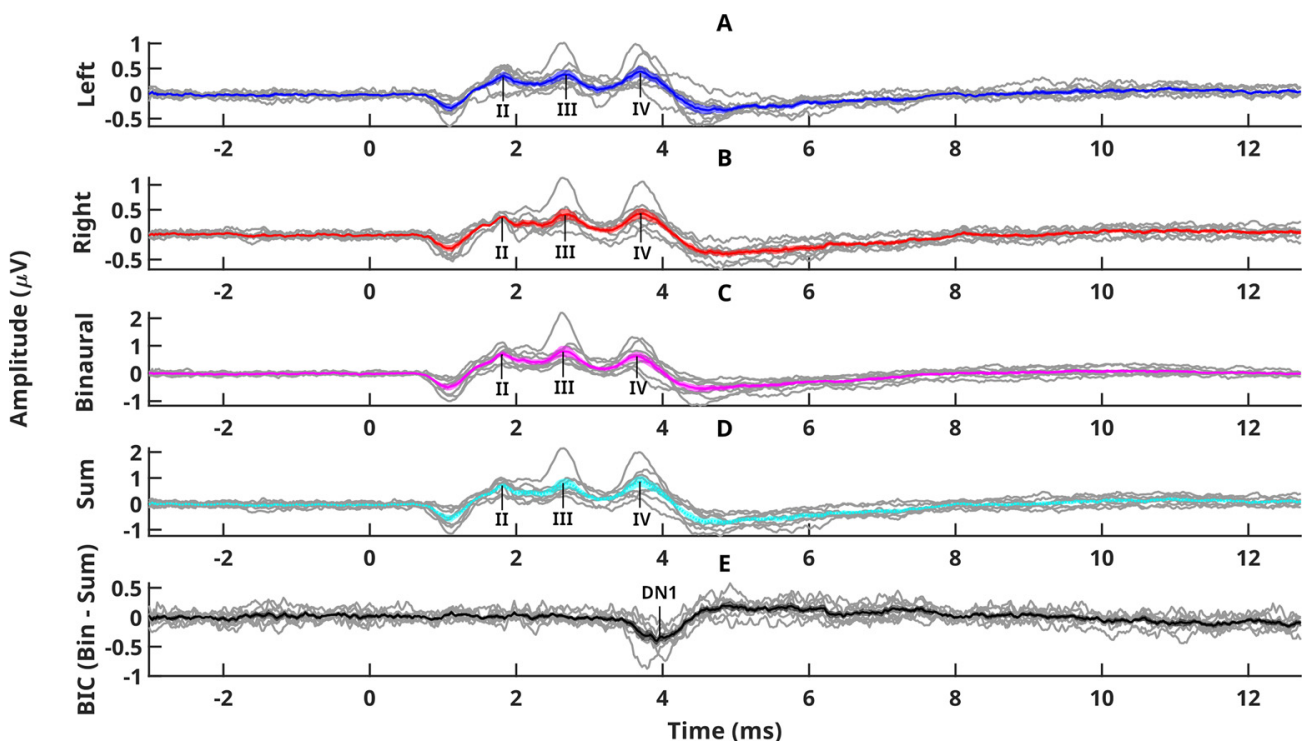

Figure 1. ABR waveforms for all eight monkeys. A, B, Averaged left and right waveforms, respectively. $\boldsymbol{C}$, Binaural signal with and ITD of $0 \mu \mathrm{s}$. $\boldsymbol{D}$, Sum of the two monaural responses. $\boldsymbol{E}$, BIC. The mean and SEM are shown by the colored line and the shaded area around the line. The gray lines show the data from each individual monkey. Different peaks of the waveforms are indicated.

increases with ITD. Figure 2E/F shows DN1 peak amplitude and latency vs ITD. Figure $2 E$ shows normalised DN1 amplitudes and Figure $2 F$ shows DN1 latency as a function of ITD. Grey lines show data from individual monkeys, while the black line shows the mean. On average, BIC DN1 was reduced $\sim 10 \%$ with $\pm 500 \mu$ s and $\sim 50 \%$ by $\pm 1000 \mu$ s ITD. Increases to $\pm 1500 \mu$ s did not produce further reductions in DN1. Latencies of DN1 approximately followed the ITD.

Figure 3 compares monkey data to several rodents (Benichoux et al., 2018), the domestic cat (Ungan et al., 1997), and human data (Riedel and Kollmeier, 2006).
Different species are indicated by colour. Figure $3 A$ displays DN1 amplitude vs ITD for eight species. Each line shows the average of all measurements for a particular species (from Benichoux et al. (2018)). To quantify these curves, a Gaussian function was fit to the data for each individual animal. The width of the fitted curves $(\sigma)$ is plotted in Figure $3 B$ as a function of average maximum ITD experienced by that species.

The data from six species (excluding cat and human) were subjected to a one-way ANOVA, which revealed no significant differences in the width $(\sigma)$ across species $\left(F_{(5,33)}=0.57, p=0.722\right)$. The width of the curve for the cat
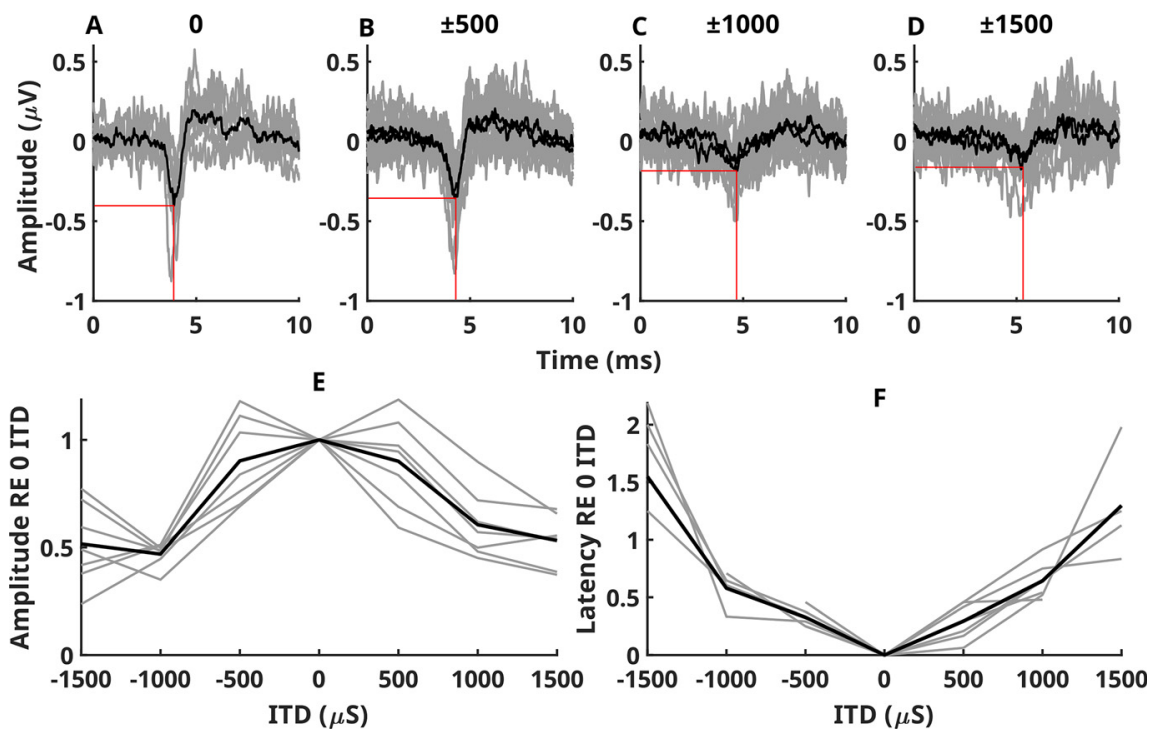

Figure 2. $\boldsymbol{A}-\boldsymbol{D}$, Raw BIC data for each measured ITD. Gray lines show measurements from individual monkeys and black lines show the mean. $\boldsymbol{E}, \boldsymbol{F}$, DN1 peak amplitude and latency versus ITD. $\boldsymbol{E}$, Normalized DN1 amplitudes. $\boldsymbol{F}$, DN1 latency as a function of ITD. Gray lines show data from individual monkeys, while the black line shows the mean. 

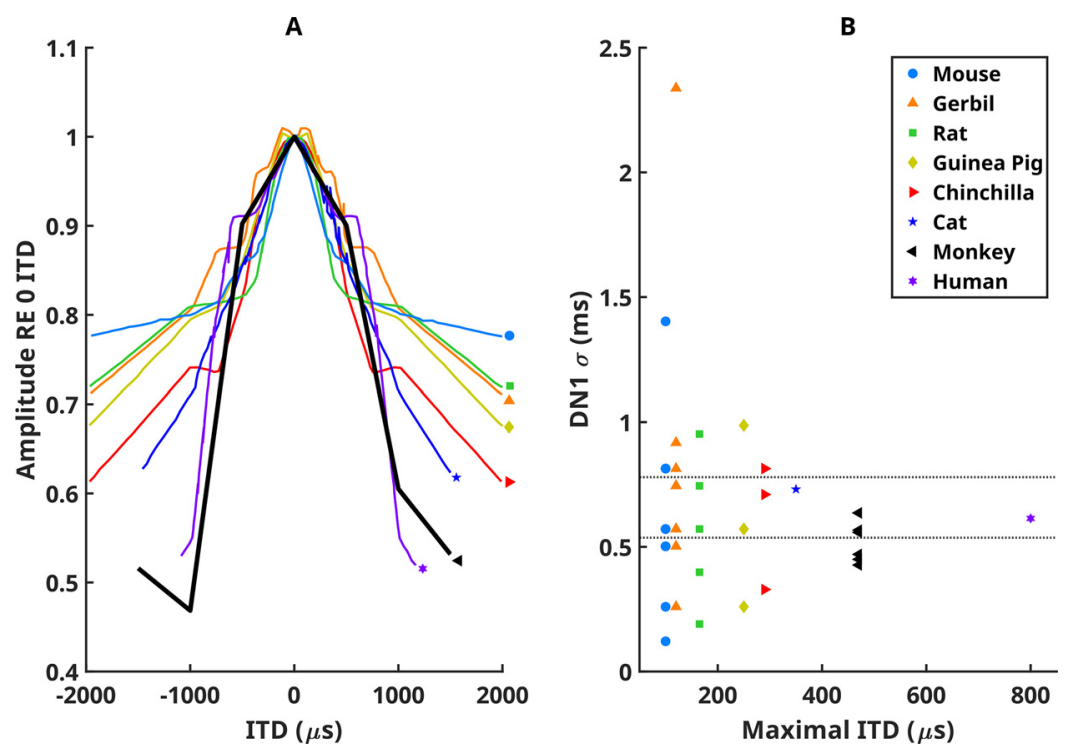

Figure 3. Monkey data compared to several rodent species from Benichoux et al. (2018), the domestic cat from Ungan et al. (1997), and human data from Riedel and Kollmeier (2006). Different species are indicated by different colors. $\boldsymbol{A}$, Normalized mean DN1 amplitude versus ITD for eight species. The width of a Gaussian function fitted to these data is plotted in $\boldsymbol{B}$ as a function of average maximum ITD experienced by each species.

and human were based on across-subject averages from other publications and thus were not included in the ANOVA statistical analysis. However, the $95 \%$ confidence interval calculated from the six species (Fig. $3 B$, dotted lines), bounds the cat and human data, indicating that humans and cats are not significantly different from the other species. The linear regression of the width $(\sigma)$ of the DN1 versus ITD curves on the magnitude of the ITD across all eight species plotted in Figure $3 B$ was not significant $\left(r^{2}=0.018, N=41\right)$. This indicates that the function relating the amplitude of DN1 to ITD does not depend on the maximum ITD magnitude experienced.

\section{Discussion}

While BIC DN1 has been examined in several mammals, many of these studies only computed DN1 for a single ITD value of $0 \mu \mathrm{s}$. Important clues to the brainstem mechanisms that produce the $\mathrm{BIC}$ can be gleaned from the function relating DN1 amplitude and latency to changes in ITD. Changes in latency can be used to test hypotheses based on existing models of brainstem ITD processing [specifically the Jeffress (1948) model], while changes in amplitude can be used to test alternative models of ITD processing based on synaptic interaction of excitation and inhibition (Ungan et al., 1997; Riedel and Kollmeier, 2006; Benichoux et al., 2018).

To date, the consensus appears to be that the DN1 latency versus ITD data are not supportive of a Jeffress-like model of ITD processing. To be consistent with Jeffress, the latency of DN1 should change at $\sim 1 / 2$ the imposed ITD, but the majority of studies show DN1 latency changing at approximately the same magnitude as the ITD (see Laumen et al., 2016). DN1 is therefore unlikely to be elicited through the brainstem circuit comprising the MSO, which has traditionally been assigned the responsibility of encoding the ITD cues.

The bulk of the current evidence supports the hypothesis that the LSO, not the MSO, is the source of the BIC.
Traditionally, the LSO has been thought to be the brainstem circuit responsible for encoding the ILD cue to location (Tollin, 2003; Owrutsky et al., 2021). Recently, Benichoux et al. (2018) showed that the function relating the amplitude of BIC DN1 to ITD was statistically indistinguishable across several rodent species, including in two species that do not have a binaurally functional MSO (mice and rats). Benichoux et al. (2018) examined this data using a computational model of the LSO similar to those suggested earlier by Ungan et al. (1997) and Riedel and Kollmeier (2006), and more recently formalized by Ashida et al. $(2016,2017)$. A more direct study demonstrated that DN1 derived from the multiunit spiking of LSO neurons recorded in vivo exhibits the same ITD dependence as the simultaneously measured ABR-derived BIC, while DN1 derived from MSO neurons recorded in vivo does not (Tolnai and Klump, 2020). Collectively, these results support the hypothesis that the LSO, not the MSO, produces the DN1 component of the BIC ABR.

In this study, we added results from rhesus macaques, which were chosen as a link between smallsized rodents and humans. Statistical analysis of the width of the DN1 amplitude versus ITD curves (Fig. 3B) indicates no significant differences between the species tested. This result provides evidence that the brainstem circuit that produces the BIC is conserved across these species despite their vastly different head sizes, and the nearly one order of magnitude differences in available ITDs. This further supports the notion that BIC DN1 is not linked to the range of naturally occurring ITDs for any given species.

Provided the monkeys were deeply anaesthetized, DN1 was identifiable in one measurement session but required 3000 signal repetitions per measurement condition. In the study of rodents by Benichoux et al. (2018), reliable DN1 
was measured with 500 stimulus repetitions per condition, while human BIC studies have required between $\sim 8000$ and 13,000 repetitions (Riedel and Kollmeier, 2006; Sammeth et al., 2020). Thus, while all species tested so far exhibit the same BIC DN1 characteristics, acquisition of reliable measures of DN1 across these species is quite different.

There are important differences between humans, macaques, and the smaller mammals that might provide an explanation for the apparent differences in the BIC's measurability: (1) in animals with larger heads the neural generators of the $\mathrm{BIC}$ are farther from the electrodes and (2) in different species the volume of the MNTB (Medial nucleus of the trapezoid body)-LSO nuclei is slightly smaller relative to the total auditory brainstem volume (Glendenning and Masterton, 1998). For the most part, the relative size of the MNTB-LSO complex is fairly consistent except for the very smallest species (mice and bats) where the MNTB-LSO is relatively large, at least in the 53 mammalian species examined by Glendenning and Masterton (1998). This suggests that the size of the MNTB-LSO might not be able to explain the reduction in reliability of evoked DN1 in the Macaque and human relative to other species, and thus the size of the head may be more significant.

Further measurements that quantify the differences in BIC measurability will be necessary to further explore these issues. For example, it is possible that the total numbers of MNTB-LSO neurons in a given species is more important that the relative volume of these nuclei. However, detailed studies of numbers of neurons comprising all auditory brainstem nuclei in many mammalian species are lacking, and none conducted by the same set of researchers in the same laboratory, such as the Glendenning and Masterton (1998) database.

In conclusion, we examined the BIC in rhesus macaques, a primate with a head size intermediate between small rodents and humans. We found that the overall form of the function relating BIC DN1 amplitude to ITD appeared statistically indistinguishable from smaller rodents, suggesting that the brainstem circuitry that produces the BIC is largely conserved across these taxa. We also note apparent differences in the measurability of the $\mathrm{BIC}$ in different species. We suggest that head size is the most likely explanation for these differences, however further measurements that quantify measurability in different species will be necessary to prove this.

\section{References}

Ashida G, Kretzberg J, Tollin DJ (2016) Roles for coincidence detection in coding amplitude-modulated sounds. PLoS Comput Biol 12:e1004997.
Ashida G, Tollin DJ, Kretzberg J (2017) Physiological models of the lateral superior olive. PLoS Comput Biol 13:e1005903.

Benichoux V, Ferber A, Hunt S, Hughes E, Tollin D (2018) Across species "natural ablation" reveals the brainstem source of a noninvasive biomarker of binaural hearing. J Neurosci 38:8563-8573.

Delb W, Strauss DJ, Hohenberg G, Plinkert PK (2003) The binaural interaction component (BIC) in children with central auditory processing disorders (CAPD). Int J Audiol 42:401-412.

Dobie RA, Berlin Cl (1979) Binaural interaction in brainstem-evoked responses. Arch Otolaryngol 105:391-398.

EIMoazen D, Sobhy O, Abdou R, AbdelMotaleb HA (2020) Binaural interaction component of the auditory brainstem response in children with autism spectrum disorder. Int J Pediatr Otorhinolaryngol 131:109850.

Furst M, Eyal S, Korczyn AD (1990) Prediction of binaural click lateralization by brainstem auditory evoked potentials. Hear Res 49:347-359.

Glendenning KK, Masterton RB (1998) Comparative morphometry of mammalian central auditory systems: variation in nuclei and form of the ascending system. Brain Behav Evol 51:59-89.

Gunnarson AD, Finitzo T (1991) Conductive hearing loss during infancy: effects on later auditory brain stem electrophysiology. J Speech Hear Res 34:1207-1215.

Jeffress LA (1948) A place theory of sound localization. J Comp Physiol Psychol 41:35-39.

Jewett DL, Romano MN, Williston JS (1970) Human auditory evoked potentials: possible brain stem components detected on the scalp. Science 167:1517-1518.

Laumen G, Ferber AT, Klump GM, Tollin DJ (2016) The physiological basis and clinical use of the binaural interaction component of the auditory brainstem response. Ear Hear 37:e276e290.

Owrutsky Z, Benichoux V, Tollin DJ (2021) Binaural hearing by the auditory brainstem - joint coding of interaural level and time differences by the lateral superior olive. In: Binaural hearing. Springer handbook of auditory research (Litovsky R, Goupell M, Popper A, Fay R, eds), pp 113-144. New York: Springer.

Riedel H, Kollmeier B (2006) Interaural delay-dependent changes in the binaural difference potential of the human auditory brain stem response. Hear Res 218:5-19.

Sammeth CA, Greene NT, Brown AD, Tollin DJ (2020) Normative study of the binaural interaction component of the human auditory brainstem response as a function of interaural time differences. Ear Hear 42:629-643.

Tollin DJ (2003) The lateral superior olive: a functional role in sound source localization. Neuroscientist 9:127-143.

Tolnai S, Klump GM (2020) Evidence for the origin of the binaural interaction component of the auditory brainstem response. Eur $\mathrm{J}$ Neurosci 51:598-610.

Ungan P, Yağcioğlu S, Ozmen B (1997) Interaural delay-dependent changes in the binaural difference potential in cat auditory brainstem response: implications about the origin of the binaural interaction component. Hear Res 106:66-82.

Van Yper LN, Vermeire K, De Vel EFJ, Beynon AJ, Dhooge IJM (2016) Age-related changes in binaural interaction at brainstem level. Ear Hear 37:434-442. 